\title{
ZraP is a periplasmic molecular chaperone and a repressor of the zinc-responsive two-component regulator ZraSR
}

\author{
Corinne APPIA-AYME, Andrea HALL, Elaine PATRICK, Shiny RAJADURAI, Thomas A. CLARKE ${ }^{1}$ and Gary ROWLEY ${ }^{1}$ \\ School of Biological Sciences, University of East Anglia, Norwich Research Park, Norwich NR4 7TJ, U.K.
}

\begin{abstract}
The bacterial envelope is the interface with the surrounding environment and is consequently subjected to a barrage of noxious agents including a range of compounds with antimicrobial activity. The ESR (envelope stress response) pathways of enteric bacteria are critical for maintenance of the envelope against these antimicrobial agents. In the present study, we demonstrate that the periplasmic protein ZraP contributes to envelope homoeostasis and assign both chaperone and regulatory function to ZraP from Salmonella Typhimurium. The ZraP chaperone mechanism is catalytic and independent of ATP; the chaperone activity is dependent on the presence of zinc, which is shown to be responsible for the stabilization of an oligomeric ZraP complex.
\end{abstract}

Furthermore, ZraP can act to repress the two-component regulatory system ZraSR, which itself is responsive to zinc concentrations. Through structural homology, ZraP is a member of the bacterial CpxP family of periplasmic proteins, which also consists of $\mathrm{CpxP}$ and Spy. We demonstrate environmental coexpression of the CpxP family and identify an important role for these proteins in Salmonella's defence against the cationic antimicrobial peptide polymyxin B.

Key words: chaperone, CpxP, polymyxin B, Salmonella, Spy, ZraP.

\section{INTRODUCTION}

Integration of environmental signals into transcriptional networks is critical for bacterial adaptation, but the mechanisms to accomplish this are complex. In Gram-negative bacteria, the message needs to be transduced across two membranes as well as the periplasm before reaching the cytoplasm where transcription is initiated. Environments or stresses which cause damage to the outer membrane, disrupting periplasmic homoeostasis, leads to induction of the ESR (envelope stress response). The response to envelope damage through the ESR often results in an increase in transcription of chaperones, proteases or isomerases, to repair or replace the damage caused. The ESR of Salmonella Typhimurium and related bacterial species includes at least five partially overlapping pathways that are regulated by the alternative sigma factor $\sigma^{\mathrm{E}}$ (RpoE), the two-component systems BaeSR and CpxRA, the Rcs phosphorelay system and the phage shock response [1].

CpxARP provides an intriguing model system for how environmental signal integration occurs. CPxAR is a twocomponent regulatory system important for responding to a number of environmental conditions that disrupt the envelope of Gram-negative bacteria (for a review see [1]). CpxP was identified as a CpxR-regulated gene in a global screen for CpxRregulated loci and combats the toxicity of envelope protein overproduction [2]. Overexpression of $\mathrm{CpxP}$ prevents activation of the Cpx pathway, and this inhibitory mechanism is dependent on the presence of the histidine kinase CpxA [3]. Evidence for direct binding of $\mathrm{CpxP}$ to $\mathrm{CpxA}$ has now been elucidated [4] although previously suggested by protein engineering, with only an N-terminal domain required for $\mathrm{Cpx}$ inhibition [5]. $\mathrm{CpxP}$ is a periplasmic adaptor protein with dual inhibitory and weak chaperone functions [6,7]. During envelope damaging conditions, CpxP is titrated away from CpxA by misfolded proteins such as PapE and presents these to the periplasmic protease DegP for proteolysis [3,8]. In 2011, two independent studies solved the protein structure of $\mathrm{CpxP}$, which forms an antiparallel dimer [9,10]. Zhou et al. [10] also present direct biochemical evidence for $\mathrm{CpxP}$ chaperone activity; $\mathrm{CpxP}$ prevents aggregation of denatured citrate synthase but cannot recover activity of the denatured protein.

Enteric bacteria such as Escherichia coli and Salmonella also possess CpxP family homologues, which include Spy and ZraP, although the protein sequence identity between ZraP and Spy, and ZraP and CpxP is only 13 and $12 \%$ respectively. Spy is a periplasmic protein, first identified as an abundant protein in spheroplasts [11] and co-regulated by two ESR pathways, CpxAR and a second two-component regulator BaeSR [12]. The precise physiological function of Spy remains unknown; however, in two independent studies, the protein structure of Spy has been solved and closely resembles that of CpxP; it forms a stable dimer with $\mathrm{N}$ - and C-terminal LTXXQ motifs [13,14]. Through a series of elegant experiments, Quan et al. [14] have shown that Spy functions as an ATP-independent chaperone, but unlike CpxP does not appear to have a regulatory role.

ZraP contains two zinc-binding domains and specifically binds zinc over related metals [15]. In this first paper assigning a physiological role to $\mathrm{ZraP}$, the authors propose that this protein is involved in zinc homoeostasis and that the periplasmic $\mathrm{C}$ terminal domain facilitates modulation of zinc transporters such as ZntA [15]. The zinc-responsive two-component regulator, ZraSR (HydH/G) regulates zraP [16], with expression of $z \mathrm{raP}$ also dependent on RpoN $\left(\sigma^{54}\right)$ [16]. So far, no functional characterization of ZraP has been performed.

In a recent study characterizing the role of BaeSR in the response to heavy metal stress [17], we observed that loss of BaeR in response to tungstate resulted in up-regulation of ZraP and ZraSR. In the present study, we have shown for the first time that

Abbreviations used: cfu, colony-forming units; $C_{t}$, threshold cycle value; ESR, envelope stress response; LB, Luria-Bertani; MDH, malate dehydrogenase; RT, reverse transcription; qRT-PCR, quantitative RT-PCR; WT, wild-type.

Correspondence may be addressed to either of the authors (email tom.clarke@uea.ac.uk or g.rowley@uea.ac.uk). 
Table 1 Strains and plasmid used in the present study

$\mathrm{Amp}^{r} / \mathrm{Ap}^{\mathrm{R}}$, ampicillin-resistant; $\mathrm{Cm}^{r}$, chloramphenicol-resistant; $\mathrm{Km}^{r} / \mathrm{Kan}^{\mathrm{R}}$, kanamycin-resistant.

\begin{tabular}{|c|c|c|}
\hline Strain/plasmid & Description & Source \\
\hline \multicolumn{3}{|l|}{ Strain } \\
\hline TOP10 & Escherichia coli TOP10 & Invitrogen \\
\hline SL1344 & Salmonella enterica serovar Typhimurium 4/74 hisG rpsL & [33] \\
\hline GR0005 & SL1344 $\Delta$ baeR & {$[17]$} \\
\hline GR0011 & SL1344 $\Delta c p x A R \Delta$ baeR & {$[17]$} \\
\hline GR0012 & SL1344 $\Delta$ spy::kan & {$[17]$} \\
\hline GR0013 & SL1344 $\Delta$ cpxP::.cat $\Delta$ spy::kan & {$[17]$} \\
\hline GR0014 & SL1344 $\Delta$ cpxP:.:cat & {$[17]$} \\
\hline GR0015 & SL1344 $\Delta$ spy::kan $\Delta$ zraP::cat & {$[17]$} \\
\hline GR0017 & SL1344 $\Delta$ zraP.:cat & {$[17]$} \\
\hline GR0018 & SL1344 $\Delta$ zraSR::kan & {$[17]$} \\
\hline GR0035 & SL1344 $\Delta$ spy $\Delta$ cpxP.:.cat $\Delta$ zraP.:kan & {$[17]$} \\
\hline GR0144 & SL1344 $\Delta$ zraP::kan & The present study \\
\hline GR0145 & SL1344 $\Delta$ zraP::kan $\Delta c p x P: .: c a t$ & The present study \\
\hline GR0146 & SL1344 zraR6His::kan & The present study \\
\hline GR0147 & SL1344 $\Delta$ baeR zraR6His::kan & The present study \\
\hline \multicolumn{3}{|l|}{ Plasmid } \\
\hline pSUB7 & For construction of $\mathrm{C}$-terminal $\mathrm{His}_{6}$ tags on the chromosome, pGP704 derivative $\left(\mathrm{Kan}^{\mathrm{R}}\right.$ ) & [21] \\
\hline pKD3 & pANTS $\gamma$ derivative $\left(\mathrm{Cm}^{r}\right)$ & {$[18]$} \\
\hline pKD4 & pANTS $\gamma$ derivative $\left(\mathrm{Km}^{r}\right)$ & {$[18]$} \\
\hline pKD46 & pBAD18 derivative $\left(\mathrm{Amp}^{r}\right)$ & [18] \\
\hline рCP20 & Temperature-sensitive replication and thermal induction of FLP synthesis $\left(\mathrm{Ap}^{\mathrm{R}}, \mathrm{Cm} \mathrm{m}^{\mathrm{r}}\right)$ & [20] \\
\hline $\mathrm{pBAD} / \mathrm{Myc}-\mathrm{His}$ & C-Terminal His 6 Tags, $\left(A p^{R}\right)$ & Invitrogen \\
\hline pZraPHis & zraP gene in $\mathrm{pBaD} / \mathrm{Myc}-\mathrm{His}$ expression plasmid in phase with the $\mathrm{His}_{6} \operatorname{tag}\left(\mathrm{Ap}^{\mathrm{R}}\right)$ & The present study \\
\hline $\mathrm{pBaeR}$ & baeR gene in $\mathrm{pBaD} / \mathrm{Myc}-\mathrm{His}$ expression plasmid without the $\mathrm{His}_{6} \operatorname{tag}\left(\mathrm{Ap} \mathrm{p}^{\mathrm{R}}\right)$ & {$[17]$} \\
\hline $\mathrm{pZraP}$ & zraP gene in $\mathrm{pBaD} / \mathrm{Myc}-\mathrm{His}$ expression plasmid without the $6 \mathrm{xH}$ is tag $\left(\mathrm{Ap}^{\mathrm{R}}\right)$ & The present study \\
\hline pSpyHis & spy gene in $\mathrm{pBaD} / \mathrm{Myc}-\mathrm{His}$ expression plasmid in phase with the $\mathrm{His}_{6}$ tag $\left(\mathrm{Ap}^{\mathrm{R}}\right)$ & The present study \\
\hline pSpy & spy gene in $\mathrm{pBaD} / \mathrm{Myc}-\mathrm{His}$ expression plasmid without the $6 \mathrm{xHis} \operatorname{tag}\left(\mathrm{Ap}^{\mathrm{R}}\right)$ & The present study \\
\hline
\end{tabular}

ZraP is a periplasmic zinc-dependent chaperone and contributes to the regulation of the two-component system ZraSR.

\section{MATERIALS AND METHODS}

\section{Bacterial strains and growth conditions}

Bacterial strains and plasmids used in the present study are described in Table 1. LB (Luria-Bertani) or LB agar plates were complemented with the appropriate antibiotic at the concentrations of $50 \mu \mathrm{g} / \mathrm{ml}$ kanamycin, $20 \mu \mathrm{g} / \mathrm{ml}$ chloramphenicol, $100 \mu \mathrm{g} / \mathrm{ml}$ ampicillin and $5 \mu \mathrm{g} / \mathrm{ml}$ tetracycline. For transcription profiling, cells were grown in LB medium to a $D_{600}$ of 1 with or without $2 \mathrm{mM}$ indole.

\section{Plasmid construction}

The zraP and spy genes were PCR-amplified from Salmonella Typhimurium SL1344 genomic DNA using the primers listed in Table 2. The PCR products were digested, ligated into the pBAD/Myc-His and transformed into E. coli strain TOP10 (Invitrogen) by electroporation. The resulting plasmids were confirmed by sequencing and are shown in Table 1 .

\section{Construction of deletion mutants}

The entire structural genes were replaced by PCR-generated antibiotic-resistance cassettes using the Lambda Red recombination system [18]. Recombinants were selected for antibiotic resistance and verified by analytical PCR. All deletions were subsequently transduced with P22-phage into a clean background [19]. For some mutants, the antibiotic gene was removed using the pCP20 plasmid $[18,20]$.

\section{Construction of $\mathrm{ZraR}^{-\mathrm{His}_{6}}$}

The C-terminal tagging of $\mathrm{ZraR}$ with the $\mathrm{His}_{6}$ epitope was performed using a modified Lambda Red method [21]. The DNA fragment to be recombined into the chromosome was PCRamplified from the pSUB7 plasmid. The successful fusion of ZraR with the $\mathrm{His}_{6}$ epitope was confirmed by PCR and Western blotting. The primers used to generate this construct are shown in Table 2.

\section{Western blot analysis}

Equal amounts of protein from Salmonella Typhimurium cell extracts were separated by SDS/PAGE (15\% gel) using a Mini Protean 3 electrophoresis system (Bio-Rad) according to the manufacturer's instructions. For immunoblotting, samples were transferred on to nitrocellulose Biodyne A membranes (Pall Corporation) using a Mini Trans-Blot Electrophoretic cell (BioRad) according to the manufacturer's instructions. The proteins were fixed with methanol and the membranes were blocked with $5 \%(\mathrm{w} / \mathrm{v})$ non-fat dried skimmed milk powder. Immobilized protein was detected using monoclonal His ${ }_{6}$ HRP (horseradish peroxidase)-conjugated antibody (1:5000 dilution). His $_{6}$-tagged ZraR was detected using a luminol-based chemiluminescent detection system (Qiagen).

\section{Protein purification $E$. coli}

TOP10 pSpyHis and pZraPHis were grown in LB medium with $50 \mu \mathrm{g} / \mathrm{ml}$ ampicillin to a $D_{600}$ of 0.5 before the addition of $0.002 \%$ and $0.02 \%$ arabinose respectively. After $4 \mathrm{~h}$ at $37^{\circ} \mathrm{C}$, the cells were harvested, resuspended in PBS containing a tablet of Complete ${ }^{\mathrm{TM}}$ protein inhibitor (Roche) and lysed by French press. After centrifugation, the His-tagged proteins were purified 
Table 2 Primers used in the present study

\begin{tabular}{|c|c|}
\hline Name & DNA sequence $\left(5^{\prime} \rightarrow 3^{\prime}\right)$ \\
\hline \multicolumn{2}{|l|}{ Mutant construction } \\
\hline baeRf & ATGACTGAATTACCCATTGATGAAAACACGCCGCGCATTTGTGTAGGCTGGAGCTGCTTC \\
\hline baeRr & CTTCCCAGCGATATCCCACCCCGTAGACCGCGCGTATAAACATATGAATATCCTCCTTAG \\
\hline spyF & ATGCGTAAACTGACTGCTCTATTTGTTGCCTCTACCCTGGGTGTAGGCTGGAGCTGCTTC \\
\hline spyR & TTCTGCCGCAGCAGGCATTTTACCTTCTTGCGCCGGGCGTCATATGAATATCCTCCTTAG \\
\hline cPXP_F & ATGCGCAAAGTTACCGCTGCTGTTATGGCCTCAACGCTGGGTGTAGGCTGGAGCTGCTTC \\
\hline cPXP_R & TTACTGGGAACGTGAGTTGCTACTACTCAATAATTTCAACCATATGAATATCCTCCTTAG \\
\hline zraP_F & GAACAATAAATCAGCTATCGCGCTAATTGCCCTCTCTCTTGTGTAGGCTGGAGCTGCTTC \\
\hline zraP_R & AGTTTCCCATACCCATGTGACCGCCGCCGCGATGATAACCCATATGAATATCCTCCTTAG \\
\hline zraSR_F & GGGGCTATTTTCCATTATGGTCATTCGCGACTATGGCCGTGTGTAGGCTGGAGCTGCTTC \\
\hline zraSR_R & GCAGCGTTTTGCGCGTAATGCCCAATTGACGGGCGGCTTCCATATGAATATCCTCCTTAG \\
\hline \multicolumn{2}{|r|}{ 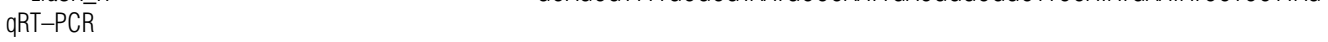 } \\
\hline RT ampD F & ATGACGAAAACCGTCCTTG \\
\hline RT ampD R & GGATCTATCGTTCCGGTGAA \\
\hline RT spy F & GCCAATGATGCAGCATAAAG \\
\hline RT spy $R$ & TAATGTCGCGAATTTGTTGC \\
\hline RT cpxPF & TAACCGAACATCAGCGTCAG \\
\hline $\mathrm{RT}$ cpxPR & CCGATGCATTGTCTCCATTT \\
\hline RT zraP F & GCAACAATGACGGTATGTGG \\
\hline RT zraPR & GCGCTGGTCTGCGTATAGTA \\
\hline RT zraR F & GTTCGGTTAATTGCCGCTAC \\
\hline RT zraR R & ATTTCAATCGCCACCACATT \\
\hline \multicolumn{2}{|l|}{ Cloning } \\
\hline Spy Nco F & TAACCATGGCCCGTAAACTGACTGCTCTATTTG \\
\hline Spy Eco R & AAGAATTCTTATTCTGCCGCAGCAGGCATTTTAC \\
\hline spyEcohis $\mathrm{R}$ & AAGAATTCTCTGCCGCAGCAGGCATTTTAC \\
\hline ZraP_Nco F & TAACCATGGTGAAACGGAACAATAAATCAG \\
\hline ZraP_Eco R & AAAAGAATTCTTTACCAGTTTCCCATACCC \\
\hline ZraP His Eco R & AAAAGAATTCCAGTTTCCCATACCCATGTG \\
\hline
\end{tabular}

from the supernatant using a HisTrap HP column on an ÄKTA FPLC (GE Healthcare).

\section{RNA and DNA extraction and quantification}

At the appropriate $D_{600}$ and before centrifugation, the cells were left on ice for a minimum of $30 \mathrm{~min}$ in a $1 \%(\mathrm{v} / \mathrm{v})$ phenol $(\mathrm{pH} 4.3), 20 \%(\mathrm{v} / \mathrm{v})$ ethanol solution to stabilize the mRNA [22]. Total RNAs were isolated using a SV Total RNA Isolation kit (Promega). Chromosomal DNA was isolated from strain Salmonella Typhimurium SL1344 using a Qiagen Genomic DNA isolation. DNA and RNA samples were quantified at $A_{260}$ and $A_{280}$ using a NanoDrop 2000c (Thermo Scientific). The quality of RNA samples were assessed by size chromatography on an Experion RNA StdSens Chip (Bio-Rad) using the Experion Automated Electrophoresis Station (Bio-Rad) according to the manufacturer's instructions.

\section{Real-time RT (reverse transcription)-PCR}

Total RNAs were first treated with Turbo DNAseFree ${ }^{\mathrm{TM}}$ from Ambion and the absence of DNA contamination was verified by PCR. Then $2 \mu \mathrm{g}$ of DNAseI-treated total RNA was reverse-transcribed from random hexamers (Invitrogen) with the Superscript II ${ }^{\mathrm{TM}}$ RT (Invitrogen) according to the manufacturer's recommendations. Gene-specific primers $\left(\sim 60^{\circ} \mathrm{C} T_{\mathrm{m}}\right)$ were designed to amplify an average product of $100 \mathrm{bp}$. The realtime PCR quantifications were realized on a 5-fold dilution of the total cDNA obtained, using the Bio-Rad CFX96 ${ }^{\mathrm{TM}}$ instrument and SensiMix ${ }^{\mathrm{TM}}$ SYBR No-ROX kit (Bioline). The real-time PCR experiments were performed in triplicate, with three independent total RNA preparations. The calculated $C_{\mathrm{t}}$ (threshold cycle value) for each gene was normalized to the $C_{\mathrm{t}}$ of the $a m p D$ gene, which expression is invariant across a large range of growth conditions.

\section{Polymyxin B assays}

Each strain was inoculated in fresh LB medium and grown to a $D_{600}$ of 0.1 at $37^{\circ} \mathrm{C}$. The cultures were challenged with polymyxin $\mathrm{B}$ (Sigma-Aldrich) at a final concentration of $4 \mu \mathrm{g} / \mathrm{ml}$, incubated at $37^{\circ} \mathrm{C}, 225 \mathrm{rev} . / \mathrm{min}$ for $1 \mathrm{~h}$ and plated on LB plates. The percentage survival was calculated as follows: survival $(\%)=$ cfu (colony-forming units) of surviving cells/cfu of initially challenged cells.

\section{Chaperone activity}

The rate of thermal aggregation of MDH (malate dehydrogenase) was recorded as described previously [14]. MDH purified from pig heart mitochondria was added to a fluorescence cuvette containing $50 \mathrm{mM}$ Hepes/ $\mathrm{NaOH}$, pH 7.5, pre-warmed to $43^{\circ} \mathrm{C}$. The final concentration of $\mathrm{MDH}$ was $213 \mathrm{nM}$ and the rate of aggregation was monitored by light scattering at a $\lambda_{\text {excitation }} / \lambda_{\text {emission }}$ of $360 \mathrm{~nm}$ with a CARY Eclipse fluorimeter equipped with a temperature controller. In addition to $\mathrm{MDH}$, the cuvette contained either 0 $198 \mathrm{nM}$ Spy or ZraP, 0-10 $\mu \mathrm{M} \mathrm{ZnCl}_{2}$ or $250 \mu \mathrm{M}$ EDTA.

\section{MDH activity assays}

Residual activity of MDH was measured through the NADH dehydrogenase activity of MDH using oxaloacetate as a substrate. Samples of $7 \mu \mathrm{M}$ MDH in $50 \mathrm{mM}$ Hepes, $\mathrm{pH} 7.4$, alone or with the addition of $70 \mu \mathrm{M}$ Spy or $70 \mu \mathrm{M}$ ZraP with $1 \mathrm{mM} \mathrm{ZnCl}_{2}$ were incubated at $45^{\circ} \mathrm{C}$ for $3 \mathrm{~h}$, then rested on ice for $10 \mathrm{~min}$. Cuvettes with $1 \mathrm{ml}$ of PBS at pH 7.4, $0.5 \mathrm{mM}$ and $0.1 \mathrm{mM} \mathrm{NADH}$ were incubated at $25^{\circ} \mathrm{C}$ and the absorbance was measured at $320 \mathrm{~nm}$ using a Hitachi U-3310 UV-visible spectrophotometer. The reaction was initiated by the addition of $2 \mu \mathrm{l}$ of incubated $\mathrm{MDH}$ to the cuvette and the rate of decrease in absorbance was 
A)

B)
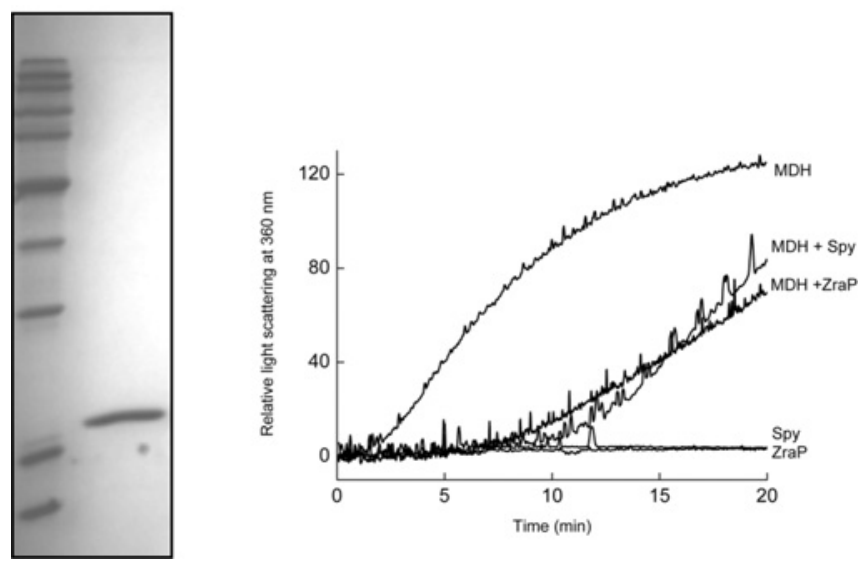

Figure 1 Chaperone activity of purified ZraP on MDH

(A) Coomassie Blue-stained SDS/PAGE of the purified ZraP-His protein. (B) Relative light scattering at $360 \mathrm{~nm}$ for $213 \mathrm{nM}$ MDH and $198 \mathrm{nM}$ Spy, $213 \mathrm{nM}$ MDH and $198 \mathrm{nM} Z \mathrm{ZraP}$, $198 \mathrm{nM}$ Spy or $198 \mathrm{nM}$ ZraP as indicated.

used to calculate the rate of NADH oxidation using an NADH absorbance coefficient of $6.22 \mathrm{mM}^{-1} \cdot \mathrm{cm}^{-1}$. The initial rate was measured using $2 \mu \mathrm{l}$ of a control sample containing $7 \mu \mathrm{M} \mathrm{MDH}$ that had not been incubated at $45^{\circ} \mathrm{C}$.

\section{Sedimentation equilibrium}

Sedimentation experiments were performed using a Beckman Optima XL-I equipped with absorbance optics. Samples of $30 \mu \mathrm{M}$ ZraP containing $50 \mathrm{mM}$ Tris/ $\mathrm{HCl}, \mathrm{pH} 7.5,50 \mathrm{mM} \mathrm{NaCl}$ and either $10 \mathrm{mM}$ EDTA, $10 \mathrm{mM} \mathrm{MgCl}{ }_{2}$ or $60 \mu \mathrm{M} \mathrm{ZnCl}_{2}$ were centrifuged at $14000 \mathrm{rev} . / \mathrm{min}$ in an An50-Ti rotor. Absorbance was monitored at $280 \mathrm{~nm}$ and scans were recorded every $5 \mathrm{~h}$ to ensure that equilibrium was achieved. Once at equilibrium, a total of five scans for each sample were recorded. A single component model was used to fit the five scans of each sample using the program ULTRASCAN [23]. Individual scans were fitted separately to determine the S.D. of the fit. The partial specific volume of ZraP was estimated from the amino acid sequence as $0.712 \mathrm{ml} / \mathrm{g}$ and a monomeric molecular mass of $13.5 \mathrm{kDa}$ was calculated from residues 27-151 predicted to remain after the removal of the signal peptide at amino acid position $26 / 27$.

\section{RESULTS}

\section{ZraP is an ATP-independent molecular chaperone}

Chaperone activity of purified ZraP (Figure 1A) was determined under experimental conditions similar to those used for Spy [14]. Incubation of $\mathrm{MDH}$ at $43^{\circ} \mathrm{C}$ causes an increase in light scattering over $20 \mathrm{~min}$ through non-specific thermal aggregation, with chaperone addition suppressing aggregation. The addition of $20 \mathrm{nM} \mathrm{ZraP}$ to $213 \mathrm{nM} \mathrm{MDH}$ suppressed the onset of light scattering for 7-10 min (Figure 1B) in a pattern similar to Spy. Spy and ZraP do not aggregate themselves under these conditions. These experiments show that purified ZraP can suppress thermal aggregation of proteins as effectively as Spy and does not require ATP to function as a chaperone.

\section{ZraP is a $\mathrm{Zn}^{2+}$-dependent catalytic chaperone}

As ZraP was originally identified as a potential zinc-dependent enzyme [15], we hypothesized that its chaperone activity would
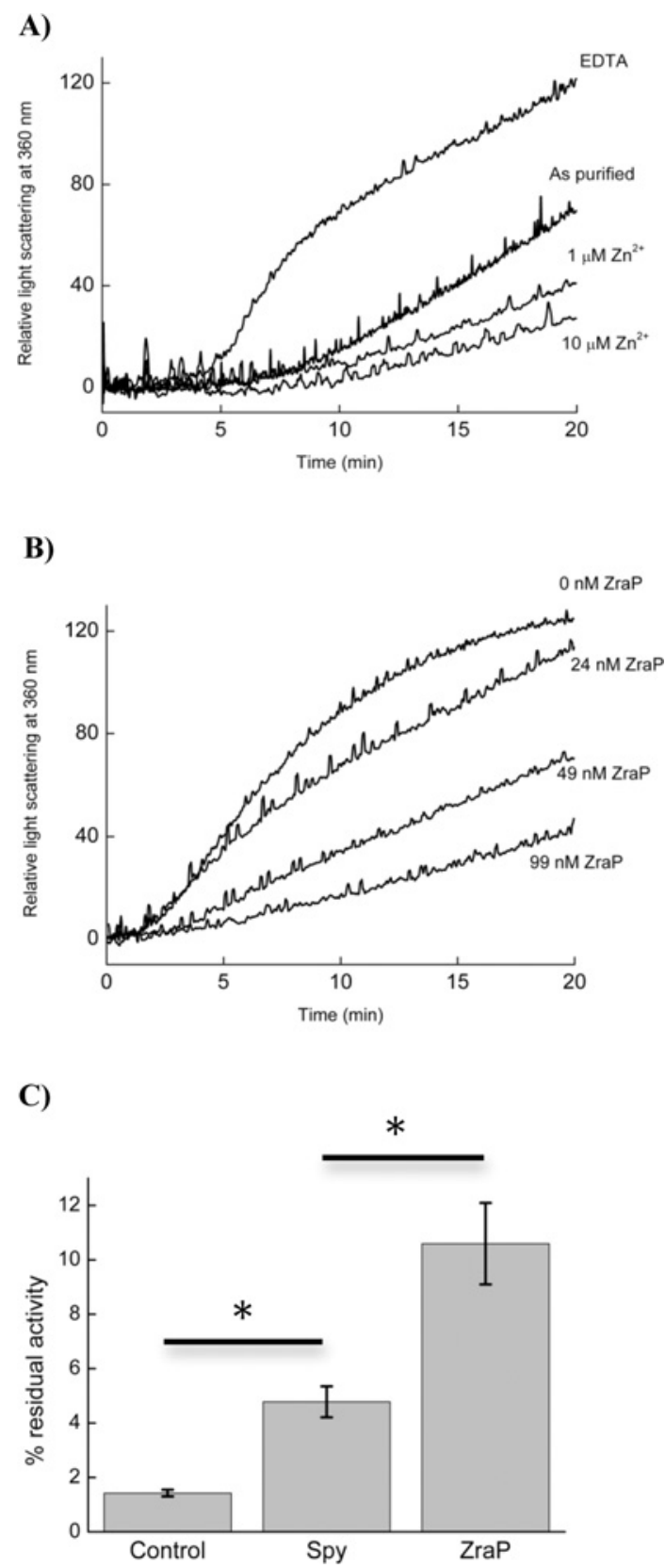

Figure 2 ZraP chaperone activity is catalytic, enhanced by zinc and protects MDH enzyme activity

(A) Thermal aggregation monitored by light scattering of $213 \mu \mathrm{M}$ isolated MDH and $198 \mathrm{nM}$ $\mathrm{ZraP}$ in the presence of $250 \mu \mathrm{M}$ EDTA, and 0,1 or $10 \mu \mathrm{M} \mathrm{ZnCl}_{2}$. (B) Thermal aggregation of $213 \mathrm{nM}$ MDH in the presence of $10 \mu \mathrm{M} \mathrm{ZnCl}_{2}$ and $0,24,49$ and $99 \mathrm{nM} \mathrm{ZraP.} \mathrm{(C)} \mathrm{Residual}$ NADH oxidase activity of $7 \mu \mathrm{M} \mathrm{MDH}$ in $50 \mathrm{mM}$ Tris/ $\mathrm{HCl}$, pH 7.5, after incubation at $45^{\circ} \mathrm{C}$ for $3 \mathrm{~h}$ in the presence of $66 \mu \mathrm{M}$ Spy or $66 \mu \mathrm{M} \mathrm{ZraP}$ and $500 \mu \mathrm{M} \mathrm{ZnCl} 2 .{ }^{*}$ Significant difference $(P<0.05$, Student's $t$ test).

be enhanced by the presence of $\mathrm{Zn}^{2+}$. To test this, purified $\mathrm{ZraP}$ was incubated with the $\mathrm{Zn}^{2+}$ chelator EDTA. ZraP treated with EDTA is not able to suppress aggregation of $\mathrm{MDH}$ as effectively as purified $\mathrm{ZraP}$, demonstrating that $\mathrm{ZraP}$ requires a metal ion to effectively function. Addition of $\mathrm{ZnCl}_{2}$ at ratios of 5 or $50 \mathrm{Zn}^{2+} / \mathrm{ZraP}$ showed that the level of $\mathrm{MDH}$ aggregation decreased with increasing concentrations of $\mathrm{Zn}^{2+}$, demonstrating that $\mathrm{Zn}^{2+}$ is required for efficient chaperone activity of $\mathrm{ZraP}$ (Figure 2A). Addition of higher concentrations of $\mathrm{ZnCl}_{2}$ did 
A

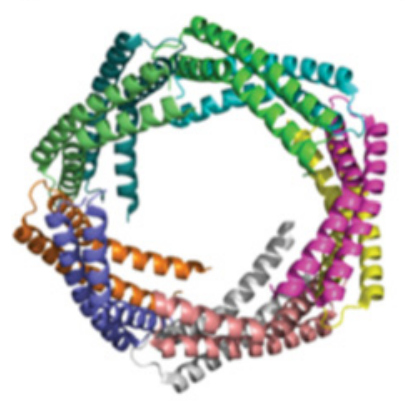

B

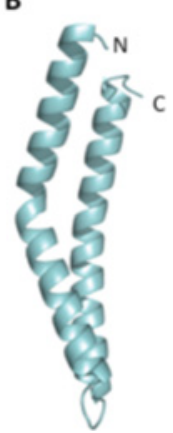

c

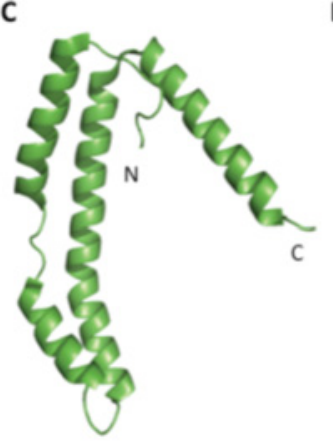

D

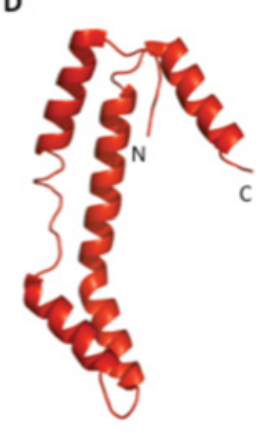

Figure 3 Cartoon representations of the known crystal structures of CpxP family members

(A) Cartoon representation of the decameric ZraP protein (PDB code 3LAY). (B) Residues Lee $4^{44}-G l u^{122}$ of the ZraP monomer. (C) Residues His ${ }^{44}-$ Lys $^{151}$ of the CpxP monomer (PDB code $3 Q Z C$ ). (D) Residues Phe ${ }^{29}-$ Thr $^{124}$ of the Spy monomer (PDB code 3039 ).

not significantly increase chaperone activity (results not shown). Control experiments showed that the presence of $\mathrm{Zn}^{2+}$ alone was not able to prevent aggregation of $\mathrm{MDH}$ (Figure 2B).

To determine whether the chaperone activity of $\mathrm{ZraP}$ is catalytic, we added $\mathrm{MDH}$ to $\mathrm{ZraP}$ at molar $\mathrm{MDH} / \mathrm{ZraP}$ ratios of $9: 1,4: 1$ or $2: 1$ (Figure 2B). Even at sub-stoichiometric concentrations, $\mathrm{ZraP}$ is able to suppress the aggregation of $\mathrm{MDH}$. $\mathrm{ZraP}$ chaperone activity is therefore catalytic and depends on a reversible interaction, which in the presence of $\mathrm{Zn}^{2+}$ is more effective than higher concentrations of Spy. We also measured the ability of both Spy and ZraP to effectively protect enzyme activity of $\mathrm{MDH}$ from thermal denaturation, using the NADH dehydrogenase activity of $\mathrm{MDH}$ in the presence of oxaloacetate. After incubation of $7 \mu \mathrm{M} \mathrm{MDH}$ for $3 \mathrm{~h}$ at $45^{\circ} \mathrm{C}$, less than $2 \%$ of the initial activity of $\mathrm{MDH}$ remained (Figure 2C). Addition of $66 \mu \mathrm{M}$ Spy to $\mathrm{MDH}$ results in a 2-fold increase in MDH activity after thermal stress, whereas a 5-fold increase in remaining activity is observed in $\mathrm{MDH}$ samples containing $66 \mu \mathrm{M}$ ZraP and $500 \mu \mathrm{M} \mathrm{ZnCl}_{2}$. These results show that, as well as preventing protein aggregation, ZraP is significantly $(P<0.05$, Student's $t$ test) more effective at protecting protein function than Spy.

\section{ZraP forms a zinc-dependent higher-order structure}

The X-ray crystal structure of ZraP was solved by the Center for Structural Genomics of Infectious Diseases and the co-ordinates are available from the RCSB Protein Data Bank (PDB code $3 \mathrm{LAY})$. ZraP is revealed as a decameric structure within which the ZraP monomer is composed of two $\alpha$-helices and residues 123-151 are disordered (Figures 3A and 3B). In CpxP (PDB code 3QZC), the residues 123-151 comprise a third $\alpha$-helix (Figure 3C); the position of this third helix in Spy (PDB code 3O39) and CpxP would interfere with the packing of a ZraPlike decameric conformation (Figures $3 \mathrm{C}$ and $3 \mathrm{D}$ ). In addition to the disordered $\mathrm{C}$-terminal residues the most significant structural differences between ZraP and the other two chaperones is midway through the N-terminal helix. This region is completely disordered in CpxP and Spy, in part due to $\mathrm{Pro}^{71}$ and $\mathrm{Pro}^{72}$ in $\mathrm{CpxP}$ and $\mathrm{Pro}^{56}$ in Spy. In ZraP there is a kink in the helix due to a broken hydrogen bond between the carbonyl of $\mathrm{Thr}^{61}$ and amide of $\mathrm{Ser}^{64}$. Thus the $\alpha$-helical pair of ZraP appears more stable than the corresponding helical pair in Spy and CpxP. The common LTXXQ motif is structurally conserved at the top of the N-terminal helix in all three proteins whereas the LTPEQ motif common to Spy and CpxP, but absent in ZraP, is located in the hinge between the middle and $\mathrm{C}$-terminal $\alpha$-helixes.
Surprisingly, on the basis of the previous study that shows that ZraP responds to zinc [15], there is no evidence for any ordered $\mathrm{Zn}^{2+}$ ions in the structure and the metal-binding prediction software TEMSP [24] failed to identify any $\mathrm{Zn}^{2+}$ binding sites within the protein structure. However, a potential $\mathrm{Zn}^{2+}$-binding motif HRGGGH is located in the amino acid sequence between 141 and 147; this region is disordered in the protein crystal structure. To investigate the possible role of $\mathrm{Zn}^{2+}$ on ZraP conformation, analytical ultracentrifugation (sedimentation equilibrium) was employed. For a single species in solution, plots of $\ln$ absorbance versus radius $^{2}-$ radius $_{(\mathrm{ref})}{ }^{2}$ would show a linear line where the gradient is proportional to the molecular mass of the single species. Figure 4 shows the sedimentation equilibrium profiles of $30 \mu \mathrm{M} \mathrm{ZraP}$ centrifuged at $15800 \mathrm{~g}$ in the presence of $10 \mathrm{mM}$ EDTA, $10 \mathrm{mM}$ $\mathrm{MgCl}_{2}$ or $60 \mu \mathrm{M} \mathrm{ZnCl}$. Fitting each absorbance profile to a single species gave an average molecular mass $\left(M_{\mathrm{w}}{ }^{\mathrm{av}}\right)$ of $73 \pm 1 \mathrm{kDa}$ for ZraP incubated with $10 \mathrm{mM}$ EDTA, and $92 \pm$ $2 \mathrm{kDa}$ for $\mathrm{ZraP}$ incubated with $10 \mathrm{mM} \mathrm{MgCl}_{2}$. The predicted mass of monomeric $\mathrm{ZraP}$ is $13.5 \mathrm{kDa}$, suggesting that in the presence of EDTA or $\mathrm{MgCl}_{2}$, the complex contains approximately 6 or $7 \mathrm{ZraP}$ monomers. Incubation of $30 \mu \mathrm{M} Z \mathrm{ZraP}$ with $60 \mu \mathrm{M} \mathrm{ZnCl}_{2}$ gave a sedimentation profile corresponding to a much larger species that could be fitted to a $M_{\mathrm{W}}{ }^{\text {av }}$ of $202 \pm 10 \mathrm{kDa}$, corresponding to an oligomer containing approximately $15 \mathrm{ZraP}$ monomers. The quality of the fits to the data is demonstrated in the top panels of Figure 4 where the residual absorbance, calculated by subtracting the experimental data from the simulated fit, is displayed. From these experiments it is impossible to distinguish whether the sedimentation profile of $\mathrm{ZnCl}_{2}$-incubated $\mathrm{ZraP}$ corresponds to a single homogenous $15 \mathrm{ZraP}$ multimer, or a heterogeneous mixture containing ZraP oligomers of 10 or 20 subunits. However, it is evident from these results that the presence of $\mathrm{Zn}^{2+}$ increases the overall size of the ZraP oligomers.

\section{Transcription of ZraP, Spy and CpxP are activated by indole}

One of the conditions known to induce spy expression in E. coli is the addition of indole to the growth medium [12]. As well as being a bacterial signalling molecule at low levels, indole can act as a biological oxidant when present in the millimolar range. As Spy and ZraP also exhibit chaperone activity akin to CpxP, combined with structural homology and cellular location, we hypothesized that functional crossover between the $\mathrm{CpxP}$ family may have also evolved. To confirm indole regulation of spy and to evaluate the transcriptional response of $c p x P$ and $z r a P$ to 


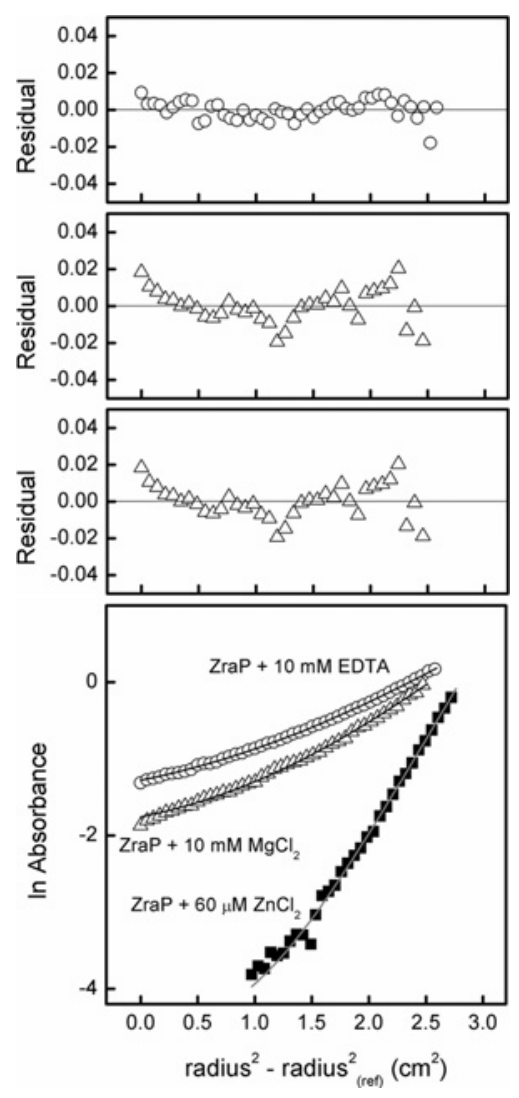

Figure 4 Sedimentation equilibrium of ZraP oligomers at $14000 \mathrm{rev} . / \mathrm{min}$

Bottom panel: absorbance profile of $30 \mu \mathrm{M} Z \mathrm{ZraP}$ measured at $280 \mathrm{~nm}$ in the presence of $10 \mathrm{mM}$ EDTA (open circles), $10 \mathrm{mM} \mathrm{MgCl}_{2}$ (open triangles) or $60 \mu \mathrm{M} \mathrm{ZnCl}_{2}$ (closed squares). Each profile was fitted to a single species non-interacting model (solid line). Absorbance data in the non-linear range of $0-0.02$ absorbance units have been excluded from the plot. Top panels: residual difference between the experimental data for each absorbance profile and fitted curves. The residual difference represents the quality of the fit between the experimental data and simulated curve.

indole, qRT-PCRs (quantitative RT-PCRs) were performed using RNA extracted from Salmonella Typhimurium strain SL1344 exposed to $2 \mathrm{mM}$ indole (Figure 5). While spy and $\operatorname{cpxP}$ were both significantly $(P<0.001$, Student's $t$ test $)$ induced 2-fold upon exposure to indole in the WT (wild-type) background, $z r a P$ was significantly $(P<0.001$, Student's $t$ test $)$ induced $\sim 35$-fold, indicating that while each of these periplasmic proteins is important for Salmonella to respond to indole, $\mathrm{zraP}$ is the most responsive to this agent. However, although expression is clearly induced by indole, mutations in any or all of the cpxP, spy and $z \mathrm{raP}$ genes in Salmonella does not affect growth or survival rate in the presence of indole under the conditions tested (results not shown).

We have previously shown that transcription of zraP and $z r a S R$ are highly up-regulated in a baeR mutant background upon addition of tungstate, and that tungstate leads to clear induction of all ESR pathways [17]. Figure 5 shows that a similar expression profile obtained for $z \mathrm{raP}$ in the presence of tungstate, is also obtained from RNA extracted from the baeR mutant upon indole addition, a known activating condition of BaeR [17], with an 80 -fold induction of $z r a P(P<0.05$, Student's $t$ test $)$ in this background plus indole compared with only a 30-fold induction in the isogenic parent strain. In E. coli, ZraSR regulates zraP expression [16]. In support of this, qRT-PCR analysis of $z \mathrm{raP}$ in a zraSR mutant background in Salmonella showed that indole
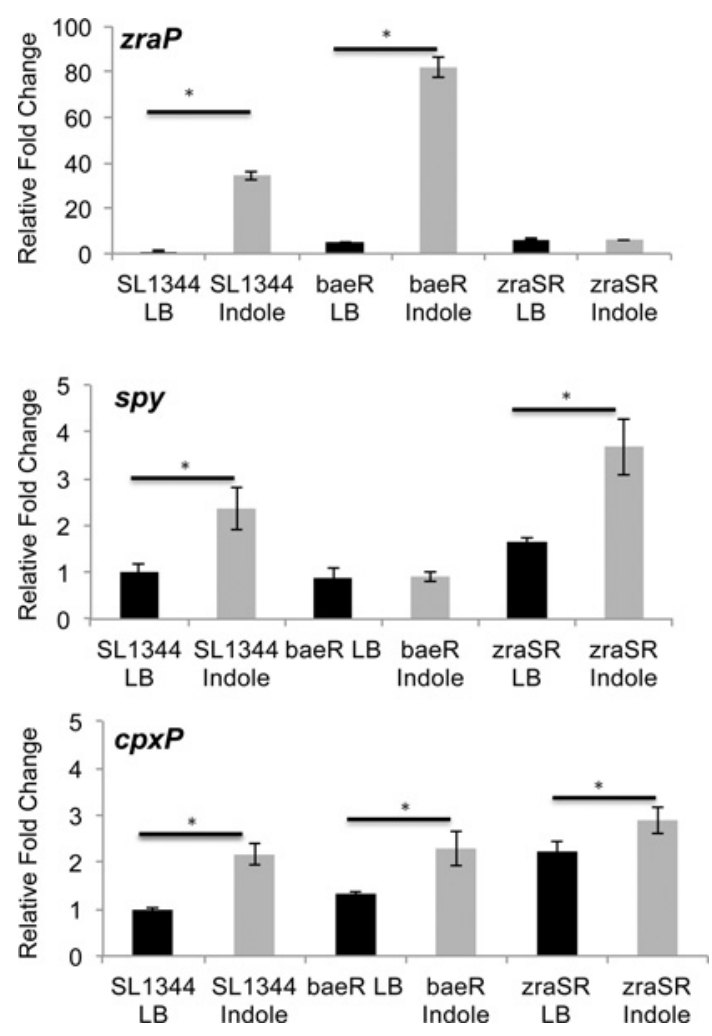

Figure 5 Expression of spy, zraP and cpxP is induced by indole

Real-time qRT-PCR of spy (black), zraP (dark grey) and cpxP (light grey) expression relative to the control gene ampD in SL1344, $\triangle$ baeR or $\triangle$ zraSR genetic backgrounds and grown in $\mathrm{LB} \pm 2 \mathrm{mM}$ indole. The induction of spy is only BaeR-dependent, while zraP induction is ZraR-dependent and is deregulated and amplified by the absence of BaeR. The indole induction of cpxP is independent of BaeR and ZraR. ${ }^{\star}$ Denotes significant difference $(P<0.05$, Student's $t$ test).

induction of $z \mathrm{raP}$ transcription was abolished (Figure 5). As anticipated, the induction of spy by indole is lost in the BaeR mutant, whereas cpxP induction by indole is similar in each of the backgrounds tested.

To investigate further the link between ZraPSR and BaeR, we confirm in Figure 6(A) that ZraR is also up-regulated at the protein level by tungstate in the absence of BaeR, whereas qRT-PCR analysis of zraP demonstrates that overexpression of BaeR results in significant repression $(P<0.05$, Student's $t$ test $)$ of $z$ raP transcription (Figure 6B). These results confirm ZraSR as a positive regulator of $z \mathrm{raP}$ activity in Salmonella, and that there is a regulatory link, either direct or indirect between the ZraPSR and BaeSR systems.

\section{ZraP regulates ZraSR activity}

Characterization of Spy and CpxP has identified overlapping and distinct functions of these proteins. They are both periplasmic chaperones, whereas only $\mathrm{CpxP}$ has an identified regulatory role. After confirming ZraP chaperone activity and regulation of $z \mathrm{raP}$ by ZraSR, we wanted to ascertain whether ZraP was more functionally similar to Spy or CpxP in terms of regulatory function. The most obvious candidate for ZraP to regulate, due to genetic location (Figure 7A) is the two-component regulator ZraSR, in a mechanism similar to that described for CpxP and CpxAR. Figure 7(B) shows that transcription of zraR in the isogenic parent background is significantly $(P<0.001$, Student's 


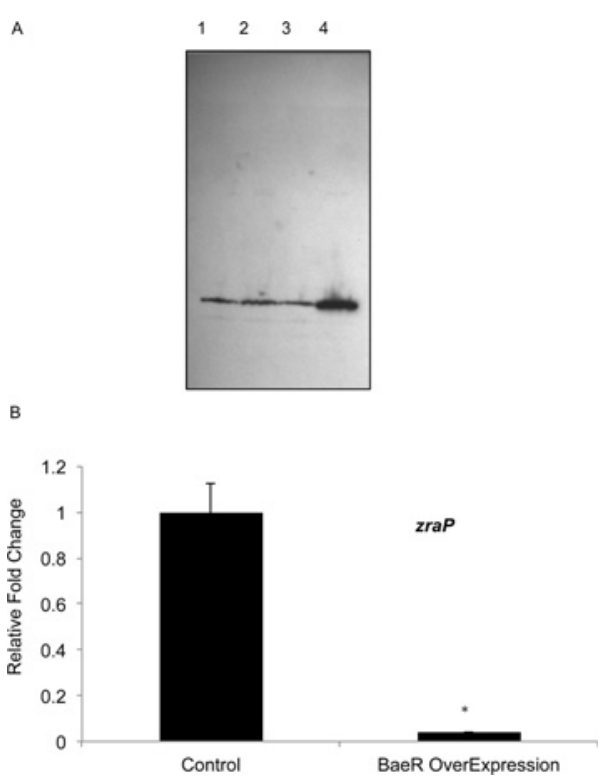

Figure 6 Expression of zraPSR is regulated by BaeSR

(A) Immunoblot of ZraR-His in Salmonella SL1344 (1 and 2) or $\Delta$ baeR (3 and 4) in LB medium ( 1 and 3 ) or LB medium $+20 \mathrm{mM}$ sodium tungstate (2 and 4 ); ZraR is more expressed in the presence of tungstate in a baeR background. (B) qRT-PCR of zraP in LB in Salmonella containing the empty pBad vector (control) or the induced pBaeR plasmid (overexpression). The overexpression of BaeR results in down-regulation of zraP. *Denotes a significant difference $(P<0.05$, Student's $t$ test).

$t$ test) induced 2-fold by the addition of indole. However, in a $z \mathrm{raP}$ mutant background zraR becomes de-regulated and its expression in LB medium is 12-fold higher $(P<0.001$, Student's $t$ test $)$ than that measured in SL1344. Addition of indole to the zraP mutant causes no significant increase in $z r a R$ expression, which is indicative of ZraSR becoming signal blind and constitutively overexpressed in the absence of ZraP. Figure 7(C) depicts the converse of Figure 7(B), with artificial overexpression of ZraP resulting in a significant $(P<0.001$, Student's $t$ test $) 2$-fold repression of $z r a R$. This clearly demonstrates that $\mathrm{ZraP}$ possesses a regulatory function and points to a mechanism more similar to that of CpxP than Spy.

\section{The CpxP family are critically required for polymyxin B resistance}

After demonstrating functional similarity between ZraP and the other CpxP family members, the question still remained as to the physiological role of these proteins in the cell. ZraP has been previously attributed to maintaining the cellular balance of zinc [15]. Transcriptomic analysis from other groups revealed that $\mathrm{ZnSO}_{4}$ induces transcription of $c p x P$, spy and zraP in E. coli [25], whereas the cationic antimicrobial peptide polymyxin B induces both spy and cpxP in Salmonella [26]. We tested the growth of Salmonella Typhimurium strain SL1344 harbouring mutations in any or all of $c p x P$, spy and $z r a P$ in the presence of $1.5 \mathrm{mM} \mathrm{ZnCl}_{2}$ (results not shown) and mutations in any or all of these loci did not affect growth or survival rate.

Polymyxin $B$ binds to the negative charge of LPS (lipopolysaccharide) and disrupts the outer membrane. Salmonella has evolved several regulatory mechanisms to resist polymyxin B and related host cationic antimicrobial peptides. We tested the ability of single, double and triple mutants of spy, cpxP and zraP to survive exposure to polymyxin B. Figure 8 shows that after 60 min exposure, each of the single mutants were significantly more sensitive to polymyxin B addition compared
A
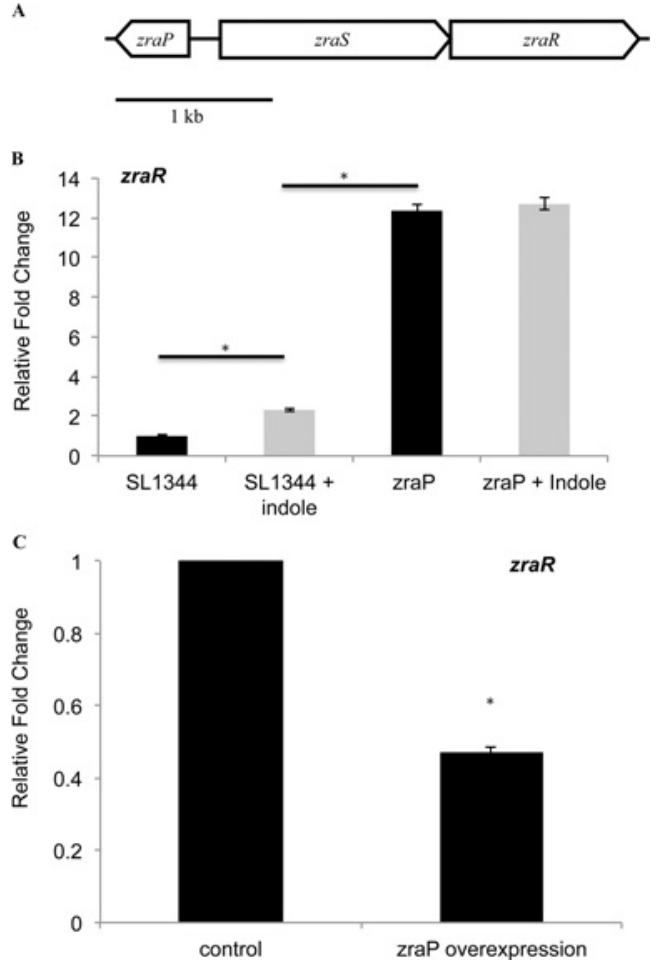

Figure 7 ZraP is a repressor of zraR expression

(A) Schematic representation of the Salmonella zraPSR locus. (B) Real-time qRT-PCR of zraR expression relative to the control gene $a m p D$, expression of $z r a R$ in $L B$ medium $\pm 2 m M$ indole in Salmonella SL1344 and a $\triangle$ zraP mutant; the absence of ZraP results in an overexpression of zraR in both conditions. (C) Expression of zraR in LB medium in Salmonella containing the empty pBad vector (control) or the induced pZraP plasmid (overexpression). The overexpression of ZraP results in down-regulation of zraR. ${ }^{\star}$ Denotes a significant difference $(P<0.05$, Student's $t$ test).

with WT, with survival of $c p x P<z r a P<\operatorname{spy}(P<0.05$, Student's $t$ test). The survival of double and triple mutations was extremely illuminating with the zraPspy double mutant much more sensitive to polymyxin B than the respective single mutants $(P<0.05$, Student's $t$ test), at a survival rate not significantly different to the single cpxP mutant, whereas a combination of loss of $c p x P$ and $z r a P$ is significantly $(P<0.05$, Student's $t$ test $)$ more sensitive to polymyxin $\mathrm{B}$ than the $c p x P$ single mutant. The triple mutant displayed the most severe phenotype, with almost no viable bacteria recovered post polymyxin B exposure, and significantly $(P<0.05$, Student's $t$ test $)$ more sensitive than the cpxPzraP double mutant. Our results indicate that, at least in the response to this polymyxin $\mathrm{B}$, there is some degree of functional overlap across this family of proteins.

\section{DISCUSSION}

As a pathogen of a wide range of both warm and cold blooded animals, Salmonella enterica encounters a multitude of environmental stresses within and out with the host, which it must survive if it is to cause a successful infection. Critical components in responding to noxious agents that cause damage to the outer membrane and/or disruption to periplasmic homoeostasis, like those generated by host macrophages including reactive oxygen and nitrogen species, are the ESR pathways. The ESR of Salmonella and related bacterial species consists of at least five overlapping pathways with each of the ESR pathways linked to a role in pathogenesis (for a review see [1]). Overlap of the 


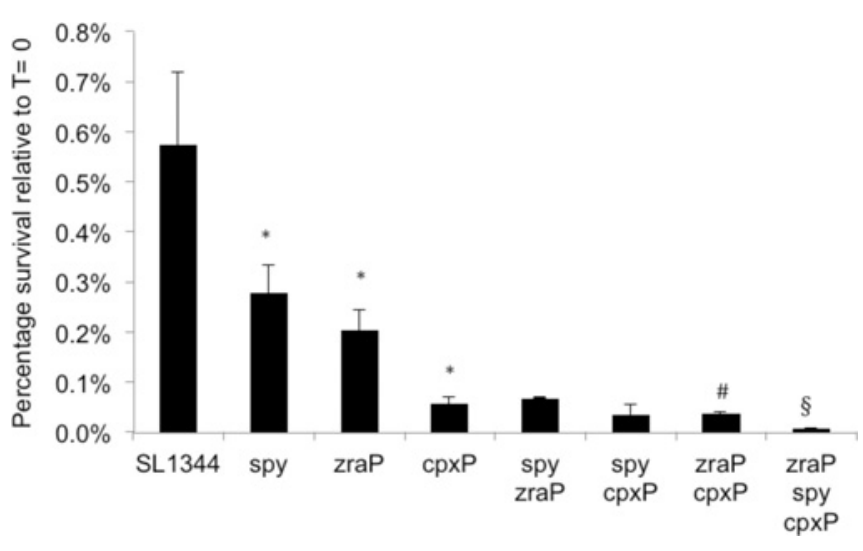

Figure 8 Spy, ZraP and CpxP contribute to Salmonella polymyxin B resistance

Percentage of survival after $1 \mathrm{~h}$ exposure to $4 \mu \mathrm{g} / \mathrm{ml}$ polymyxin B of Salmonella Typhimurium SL1344 and single, double or triple mutants in spy, zraP and cpxP genes. *Denotes a significant difference compared with SL1344, \# denotes a significant difference compared with cpxP and $\S$ denotes a significant difference compared with $\operatorname{cpxPzraP}(P<0.05$, Student's $t$ test).

ESR pathways occurs at both the inducing signals that stimulate activity and regulon members, with the prediction that genes coregulated by more than one ESR have a more important role in envelope homoeostasis.

An example of a gene co-regulated by more than one of the ESR pathways is the periplasmic protein encoding gene spy, which after investigation by a number of laboratories was recently shown to have chaperone activity in E. coli [14]. Molecular chaperones normally recognize their target substrate through an exposed region of unfolded polypeptide. Exact mechanisms have been described for how chaperones function in the cytoplasm (for a review see [27]), particularly with the DnaK machinery as a model, whereas the mechanisms and specific targets of ATP-independent molecular chaperones in the periplasm still remain relatively poorly investigated. The best characterized of the periplasmic chaperones are those such as SurA, Skp and DegP (HtrA) (for a review see [28]), all associated with targeting polypeptides to the BAM ( $\beta$-barrel assembly machinery), which is required for outer membrane biogenesis and TTS (type three secretion) in Salmonella [29]. We were intrigued by the described periplasmic chaperone activity of Spy, combined with its coregulation by at least $\mathrm{CpxR}$ and BaeR, as loss of spy in Salmonella had no significant phenotype when screened on the BioLog phenotype microarrays, which tests $\sim 2000$ different conditions (results not shown). Mutation of $d n a K$, however, results in severe temperature sensitivity and sensitivity towards exogenous stress such as $\mathrm{H}_{2} \mathrm{O}_{2}$ [30]. With this in mind and with the observation that ZraP, another periplasmic protein with weak sequence homology with Spy and CpxP, was present in the protein database, we sought to characterize the role of $\mathrm{ZraP}$ in periplasmic homoeostasis. We hypothesized that a degree of functional overlap existed between members of this small family of proteins, indicative of an important role they play in periplasmic homoeostasis of proteobacteria, which was missing from current genetic analyses.

Using a light scattering assay to determine aggregation of thermally denatured $\mathrm{MDH}$, we observed that addition of purified ZraP to the solution suppressed aggregation, at a level comparable with purified Spy, which, as indicated above, has established chaperone activity. The chaperone activity of ZraP is catalytic as aggregation is suppressed at sub-stoichiometric ratios and this activity is also ATP-independent. As ZraP had initially been assigned a role in cellular zinc homoeostasis, zinc was added to the solution to assess enzyme activity. Our observations would support the requirement of zinc for optimal chaperone activity of ZraP with sedimentation equilibrium results indicating that stabilization of high-order ZraP structures also requires zinc, although it remains to be investigated whether the oligomeric structure is itself required for chaperone activity, or indeed the regulatory role discussed below. It is worth noting, however, that a $z \mathrm{raP}$ mutant is not more sensitive to the addition or removal of zinc to the growth medium than its WT counterpart. Indeed a single mutant in $z r a P$, similar to our experience with a spy single mutant, is not more sensitive to a range of ESR conditions we tested, including high temperature, high osmolality and the presence of detergents (results not shown), except for the addition of polymyxin B. This observation led us to analyse the survival of each of the single, double and triple CpxP family member mutants in our collection for survival in the presence of polymyxin B. Each of the single mutants themselves are significantly more sensitive to polymyxin B than the isogenic parent strain, with the most dramatic difference observed with deletion of $c p x P$ and the least effect observed with loss of spy. However, certain combinations of mutants in spy, cpxP and zraP were significantly more sensitive than the single mutants, with the $\operatorname{raPcpxP}$ double mutant and the triple mutant significantly more sensitive to polymyxin B than the single cpxP mutant, and the spycpxP double mutant only just failing to pass statistical significance. Our phenotypic data indicate an overlapping requirement of Salmonella for the CpxP family members, at least under this specific condition, and suggests the combined importance of this family in maintaining envelope homoeostasis.

The regulatory activity of $\mathrm{CpxP}$ has been well studied; it acts as a repressor of CpxA auto-phosphorylation activity [3]. Despite Spy sharing structural similarities with $\mathrm{CpxP}$, no regulatory activity has yet been shown for this protein; this may be because unlike $c p x P$, spy has no regulatory candidate in close proximity and, consequently, the search for a regulatory partner is more problematic. The $\mathrm{raSR}$ locus is next to $\mathrm{raP}$ on the chromosome and is transcribed in the opposite direction, making zraSR the most obvious target to be regulated by ZraP. We show in this paper that the expression of $z r a R$ is deregulated when $\mathrm{ZraP}$ is not present and that artificial overexpression of ZraP down-regulates $z r a R$ expression, suggesting that this periplasmic chaperone can also act as a repressor of the ZraSR two-component system. We also provide further evidence to support regulatory cross-talk between the ZraPSR and BaeSR systems. Loss of BaeR leads to induction of ZraPSR, whereas overexpression of BaeR represses ZraPSR. Intriguingly a recent study that models the dynamics of the transcriptional response of $E$. coli to zinc addition [31], describes repression of baeR and we propose that this will be due to up-regulation of the zinc-responsive ZraPSR.

Although the last to be functionally characterized, we suggest that under certain stress conditions or environments, ZraP is likely to be very important for maintaining periplasmic homoeostasis. First, as shown by the qRT-PCR and microarray analysis [17], induction of $z \mathrm{raP}$ in the presence of indole and tungstate was much higher than observed for $c p x P$ and spy. Furthermore, ZraP seems to have acquired all activities of its protein family, indeed, $\mathrm{CpxP}$ has only weak chaperone activity but has periplasmic regulatory activity, Spy has no known regulatory activity but is a strong periplasmic chaperone. ZraP, however, has strong chaperone activity, comparable with, if not better than, Spy, and also has periplasmic regulatory activity through repressing zraSR. The periplasmic substrates of ZraP are yet to be identified and will form part of further biophysical and biochemical analyses of ZraP. Indeed whether ZraP substrates are specific proteins or exposed hydrophobic patches in generally misfolded or damaged proteins remains to be resolved. 
In conclusion, the results of the present study point to functional overlap across the $\mathrm{CpxP}$ family in maintaining envelope homoeostasis. Structure-function analyses are warranted to identify physiological targets of these chaperones and the mechanisms involved, and elucidate whether the conserved domains of these proteins make possible targets for novel antimicrobials. Using our triple mutant the role of the CpxP family in pathogenesis also requires elucidation on the basis of the sensitivity profile to polymyxin B. We also propose that ZraPSR contributes to the ESR of Salmonella and related enteric bacteria and the ZrasR regulon of Salmonella needs to be investigated further. In support of this, after identifying that expression of Salmonella zraSR is up-regulated during infection of pigs, zraSR was also shown to be up-regulated by other common inducers of the ESR, including temperature and osmolarity shock [32].

\section{AUTHOR CONTRIBUTION}

Corinne Appia-Ayme, Andrea Hall, Elaine Patrick, Shiny Rajadurai and Thomas Clarke performed the experiments. Corinne Appia-Ayme, Thomas Clarke and Gary Rowley designed the experiments, analysed the data and wrote the paper.

\section{ACKNOWLEDGEMENTS}

We thank Professor Andrew Johnston, Professor David Richardson and Dr Matthew Hutchings, UEA for extremely useful discussions.

\section{FUNDING}

This work was supported by the Biotechnology and Biological Sciences Research Council (U.K.) [grant number BB/G020582/1].

\section{REFERENCES}

1 Rowley, G., Spector, M., Kormanec, J. and Roberts, M. (2006) Pushing the envelope: extracytoplasmic stress responses in bacterial pathogens. Nat. Rev. Microbiol. 4, 383-394

2 Danese, P. N. and Silhavy, T. J. (1998) CpxP, a stress-combative member of the Cpx regulon. J. Bacteriol. 180, 831-839

3 Raivio, T. L., Popkin, D. L. and Silhavy, T. J. (1999) The Cpx envelope stress response is controlled by amplification and feedback inhibition. J. Bacteriol. 181, 5263-5272

4 Fleischer, R., Heermann, R., Jung, K. and Hunke, S. (2007) Purification, reconstitution, and characterization of the CpxRAP envelope stress system of Escherichia coli. J. Biol. Chem. 282, 8583-8593

5 Buelow, D. R. and Raivio, T. L. (2005) Cpx signal transduction is influenced by a conserved $\mathrm{N}$-terminal domain in the novel inhibitor CpxP and the periplasmic protease DegP. J. Bacteriol. 187, 6622-6630

6 DiGiuseppe, P. A. and Silhavy, T. J. (2003) Signal detection and target gene induction by the CpxRA two-component system. J. Bacteriol. 185, 2432-2440

7 Isaac, D. D., Pinkner, J. S., Hultgren, S. J. and Silhavy, T. J. (2005) The extracytoplasmic adaptor protein CpxP is degraded with substrate by DegP. Proc. Natl. Acad. Sci. U.S.A. 102, 17775-17779

8 Raivio, T. L., Laird, M. W., Joly, J. C. and Silhavy, T. J. (2000) Tethering of CpxP to the inner membrane prevents spheroplast induction of the cpx envelope stress response. Mol. Microbiol. 37, 1186-1197

9 Thede, G. L., Arthur, D. C., Edwards, R. A., Buelow, D. R., Wong, J. L., Raivio, T. L. and Glover, J. N.M. (2011) Structure of the periplasmic stress response protein CpxP. J. Bacteriol. 193, 2149-2157

10 Zhou, X., Keller, R., Volkmer, R., Krauss, N., Scheerer, P. and Hunke, S. (2011) Structural basis for two-component system inhibition and pilus sensing by the auxiliary CpxP protein. J. Biol. Chem. 286, 9805-9814
11 Hagenmaier, S., Stierhof, Y. D. and Henning, U. (1997) A new periplasmic protein of Escherichia coli which is synthesized in spheroplasts but not in intact cells. J. Bacteriol. 179, 2073-2076

12 Raffa, R. G. and Raivio, T. L. (2002) A third envelope stress signal transduction pathway in Escherichia coli. Mol. Microbiol. 45, 1599-1611

13 Kwon, E., Kim, D. Y., Gross, C. A., Gross, J. D. and Kim, K. K. (2010) The crystal structure Escherichia coli Spy. Protein Sci. 19, 2252-2259

14 Quan, S., Koldewey, P., Tapley, T., Kirsch, N., Ruane, K. M., Pfizenmaier, J., Shi, R., Hofmann, S., Foit, L., Ren, G. et al. (2011) Genetic selection designed to stabilize proteins uncovers a chaperone called Spy. Nat. Struct. Mol. Biol. 18, 262-269

15 Noll, M., Petrukhin, K. and Lutsenko, S. (1998) Identification of a novel transcription regulator from Proteus mirabilis, PMTR, revealed a possible role of YJAI protein in balancing zinc in Escherichia coli. J. Biol. Chem. 273, 21393-21401

16 Leonhartsberger, S., Huber, A., Lottspeich, F. and Böck, A. (2001) The hydH/G genes from Escherichia coli code for a zinc and lead responsive two-component regulatory system. J. Mol. Biol. 307, 93-105

17 Appia-Ayme, C., Patrick, E., Sullivan, M. J., Alston, M. J., Field, S. J., Abuoun, M., Anjum, M. F. and Rowley, G. (2011) Novel inducers of the envelope stress response BaeSR in Salmonella Typhimurium: BaeR Is critically required for tungstate waste disposal. PLoS ONE 6, e23713

18 Datsenko, K. A. and Wanner, B. L. (2000) One-step inactivation of chromosomal genes in Escherichia coli K-12 using PCR products. Proc. Natl. Acad. Sci. U.S.A. 97, 6640-6645

19 Gemski, Jr, P. and Stocker, B. A. (1967) Transduction by bacteriophage P22 in nonsmooth mutants of Salmonella Typhimurium. J. Bacteriol. 93, 1588-1597

20 Cherepanov, P. P. and Wackernagel, W. (1995) Gene disruption in Escherichia coli: TcR and $\mathrm{KmR}$ cassettes with the option of Flp-catalyzed excision of the antibiotic-resistance determinant. Gene 158, 9-14

21 Uzzau, S., Figueroa-Bossi, N., Rubino, S. and Bossi, L. (2001) Epitope tagging of chromosomal genes in Salmonella. Proc. Natl. Acad. Sci. U.S.A. 98, 15264-15269

22 Tedin, K. and Blasi, U. (1996) The RNA chain elongation rate of the lambda late mRNA is unaffected by high levels of ppGpp in the absence of amino acid starvation. J. Biol. Chem. 271, 17675-17686

23 Demeler, B. (2005) UltraScan - a comprehensive data analysis software package for analytical ultracentrifugation experiments. In Modern Analytical Ultracentrifugation: Techniques and Methods (Scott, D. J., Harding, S. E. and Rowe, A. J., eds), pp. 210-229, Royal Society of Chemistry, U.K.

24 Zhao, W., Xu, M., Liang, Z., Ding, B., Niu, L., Liu, H. and Teng, M. (2011) Structure-based de novo prediction of zinc-binding sites in proteins of unknown function. Bioinformatics 27, 1262-1268

25 Graham, A. I., Hunt, S., Stokes, S. L., Bramall, N., Bunch, J., Cox, A. G., Mcleod, C. W. and Poole, R. K. (2009) Severe zinc depletion of Escherichia coli: roles for high affinity zinc binding by ZinT, zinc transport and zinc-independent proteins. J. Biol. Chem. 284 18377-18389

26 Bader, M. W., Navarre, W. W., Shiau, W., Nikaido, H., Frye, J. G., McClelland, M., Fang, F. C. and Miller, S. I. (2003) Regulation of Salmonella typhimurium virulence gene expression by cationic antimicrobial peptides. Mol. Microbiol. 50, 219-230

27 Houry, W. A. (2001) Chaperone-assisted protein folding in the cell cytoplasm. Curr. Protein Pept. Sci. 2, 227-244

28 Merdanovic, M., Clausen, T., Kaiser, M., Huber, R. and Ehrmann, M. (2011) Protein quality control in the bacterial periplasm. Annu. Rev. Microbiol. 65, 149-168

29 Fardini, Y., Trotereau, J., Bottreau, E., Souchard, C., Velge, P. and Virlogeux-Payant, I. (2009) Investigation of the role of the BAM complex and SurA chaperone in outer-membrane protein biogenesis and type III secretion system expression in Salmonella. Microbiology 155, 1613-1622

30 Delaney, J. M. (1990) Requirement of the Escherichia coli dnaK gene for thermotolerance and protection against $\mathrm{H}_{2} \mathrm{O}_{2}$. J. Gen. Microbiol. 136, 2113-2118

31 Graham, A. I., Sanguinetti, G., Bramall, N., McLeod, C. W. and Poole, R. K. (2012) Dynamics of a starvation-to-surfeit shift: A transcriptomic and modelling analysis of the bacterial response to zinc reveals transient behaviour of the Fur and SoxS regulators. Microbiology, 158, 284-292

32 Huang, Y., Leming, C. L., Suyemoto, M. and Altier, C. (2007) Genome-wide screen of Salmonella genes expressed during infection in pigs, using in vivo expression technology. Appl. Environ. Microbiol. 73, 7522-7530

33 Hoiseth, S. K. and Stocker, B. A. (1981) Aromatic-dependent Salmonella typhimurium are non-virulent and effective as live vaccines. Nature 291, 238-239 